\title{
The number of positive solutions of a non-linear problem with discontinuous non-linearity
}

\author{
Jacques Douchet \\ Ecole Polytechnique Fédérale de Lausanne, 61 avenue de Cour, 1007 Lausanne, \\ Switzerland
}

(Ms received 5 February 1981)

\section{Synopsis}

For the non-linear problem

$$
\left\{\begin{aligned}
-u^{\prime \prime}(x) & =\lambda f(u(x)) \text { for } 0<x<1 \\
u(0) & =u(1)=0
\end{aligned}\right.
$$

where $f$ is a discontinuous function at 1 , we show that the number of non-trivial positive solutions, for a given real number $i \geqq 0$, is related to the graph of a continuous function $\mathrm{g}$. Then, by studying the function $g$ it is possible in some special cases to give, for any $\lambda \geqq 0$, the minimal or exact number of non-trivial positive solutions.

\section{Introduction}

We consider the non-linear two point boundary-value problem

$$
\left\{\begin{aligned}
-u^{\prime \prime}(x) & =\lambda f(u(x)) \text { for } \quad 0<x<1 \\
u(0) & =u(1)=0
\end{aligned}\right.
$$

where $f:[0,+\infty) \rightarrow[0,+\infty)$ is given. We assume that there exist two continuously differentiable functions $h:[0,1] \rightarrow[0,+\infty)$ and $k:[1,+\infty) \rightarrow(0,+\infty)$ such that

$$
\begin{gathered}
h(0)=0, \\
h(p)>0 \text { for all } p \in(0,1], \\
h(1) \neq k(1), \\
f(p)=\left\{\begin{array}{lll}
h(p) & \text { if } p \in[0,1) \\
k(p) & \text { if } & p \in(1,+\infty) .
\end{array}\right.
\end{gathered}
$$

The value of $f$ at 1 need not be related to $h$ and $k$, but $f(1)$ should be positive. G

DEFINITION. A solution of problem $(1.1)$ is a pair $(u, \hat{\lambda}) \in C^{1}([0,1]) \times[0,+\infty)$ 
such that

$$
\begin{aligned}
& u(x) \geqq 0 \quad \text { for all } x \in[0,1], \\
& u(0)=u(1)=0, \\
& u^{\prime} \text { is absolutely continuous on }[0,1]
\end{aligned}
$$

and

$$
-u^{\prime \prime}(x)=\lambda f(u(x)) \text { for almost all } x \in[0,1] .
$$

We denote by $S$ the subset of $C^{1}([0,1]) \times[0,+\infty)$ consisting of all the solutions of problem (1.1). Since $f(0)=0$, the set of trivial solutions $\left\{(0, \lambda) \in C^{1}([0,1) \times\right.$ $[0,+\infty) \mid \lambda \geqq 0\}$ belongs to $S$. Let $S^{+}=\{(u, \lambda) \in S \mid\|u\| \neq 0\}$ where $\|u\|=$ $\max _{x \in[0,1]}|u(x)|$.

In order to prove the existence of solutions in $S^{+}$, we consider for all $n \in \mathbb{N}$ the following problem:

$$
\left\{\begin{array}{c}
-u^{\prime \prime}(x)=\lambda f_{n}(u(x)) \text { for } \quad 0<x<1 \\
u(0)=u(1)=0
\end{array}\right.
$$

where $f_{n}:[0,+\infty) \rightarrow(0,+\infty)$ is defined by $f_{n}(t)=f(t)+(1 / n)$ for all $t$ in $[0,+\infty)$. Let $S_{n}$ be the subset of $C^{1}([0,1]) \times(0,+\infty)$ consisting of all the solutions of problem $(1.1)_{(\mathrm{n})}$. Then (see $\left.[1,2,3]\right)$, we know that for any $\rho>0$, there exists a unique $\left(u_{n}, \lambda_{n}\right)$ in $S_{n}$ such that $\left\|u_{n}\right\|=\rho$. Moreover $\left(u_{n}, \lambda_{n}\right)$ has the following properties:

(1) $\lambda_{n}=g_{n}^{2}\left(\left\|u_{n}\right\|\right)$, where $g_{n}:(0,+\infty) \rightarrow(0,+\infty)$ is a continuous function defined by $g_{n}(p)=\sqrt{2} \int_{0}^{p}\left\{F_{n}(p)-F_{n}(\omega)\right\}^{-\frac{1}{2}} d \omega, F_{n}(\omega)=\int_{0}^{\omega} f_{n}(s) d s ;$

(2) $u_{n}^{\prime}(x)>0$ for all $x \in\left[0, \frac{1}{2}\right)$ and $u^{\prime}(x)<0$ for all $x \in\left(\frac{1}{2}, 1\right]$;

(3) $u_{n}\left(\frac{1}{2}\right)=\rho$ and $u_{n}^{\prime}\left(\frac{1}{2}\right)=0$;

(4) if $\rho=1$, then $\left\{x \in[0,1] \mid u_{n}(x)=1\right\}=\left\{\frac{1}{2}\right\}$;

if $\rho>1$, then there exists $x_{0} \in\left(0, \frac{1}{2}\right)$ such that $\left\{x \in[0,1] \mid u_{n}(x)=1\right\}=\left\{x_{0}, 1-x_{0}\right\}$;

(5) $u_{n}(x)=u_{n}(1-x)$ for all $x \in[0,1]$.

Now that these definitions have been given, let us state our main results.

In Section 3, we show that for any $\rho>0$ there exists a unique solution $(u, \lambda)$ in $S^{+}$such that $\|u\|=\rho$. We also state that $S^{+}$is a continuum in $C^{1}([0,1]) \times$ $[0,+\infty)$ and furthermore $S^{+}$is a continuous curve in $C^{1}([0,1]) \times[0,+\infty)$ which can be parameterized by $\|u\|$. The last result of Section 2 is that if $h(1)<k(1)$ and $\lim _{p \rightarrow+\infty} p^{-1} f(p)=0$, then there are always values of $\lambda$ for which there exist at least two distinct solutions of problem (1.1) in $S^{+}$.

In Section 3, we study the case $h^{\prime}(0)=\lim _{p \rightarrow 0^{+}} p^{-1} f(p)=\alpha>0$. We show that $\mathscr{C}=S^{+} \cup\left\{\left(0, \pi^{2} / \alpha\right)\right\}$ is a continuum in $C^{1}([0,1]) \times[0,+\infty)$ and furthermore $\mathscr{C}$ is a 
continuous curve in $C^{1}([0,1]) \times[0,+\infty)$ which can be parameterized by $\|u\|$. We also state that if $\lim _{p \rightarrow+\infty} p^{-1} f(p)=0$, then for any $\lambda \geqq \pi^{2} / \alpha$ there is at least one solution of problem (1.1) in $\mathscr{C}$. For the two following cases:

$$
\begin{aligned}
& f(p) \geqq p f^{\prime}(p) \text { for all } p \in[0,1) \cup(1,+\infty) \text { and } h(1)>k(1) \text {, } \\
& f(p) \leqq p f^{\prime}(p) \text { for all } p \in[0,1) \cup(1,+\infty) \text { and } h(1)<k(1),
\end{aligned}
$$

we give, for any $\lambda \geqq 0$, the exact number of solutions of problem (1.1) in $\mathscr{C}$. At the end of Section 3 , we give a theorem in which, for some values of $\lambda$, there exist at least three distinct solutions of problem $(1.1)$ in $\mathscr{C}$.

In Section 4, we study the case $h^{\prime}(0)=\lim _{p \rightarrow 0^{+}} p^{-1} f(p)=0$. We show the existence of a number $\hat{\lambda} \geqq 0$ such that, for any $\lambda>\hat{\lambda}$, there is at least one solution of problem (1.1) in $S^{+}$. Moreover, if $\lim _{p \rightarrow+\infty} p^{-1} f(p)=0$, there exists a positive number $\hat{\lambda}_{1}$ such that, for any $\lambda>\lambda_{1}$ there are at least two distinct solutions of problem (1.1) in $S^{+}$, for $\lambda=\lambda_{1}$ there is at least one solution in $S^{+}$and for $\lambda \in\left[0, \lambda_{1}\right)$ there is no solution in $S^{+}$. As in. Section 3 , for the case (B): $f(p) \leqq p f^{\prime}(p)$ for all $p \in[0,1) \cup(1,+\infty)$ and $h(1)<k(1)$, we give, for any $\lambda \geqq 0$, the exact number of solutions of problem (1.1) in $S^{+}$.

The problem (1.1) has already been studied in the following three articles. In [4], Laetsch studies the problem (1.1) with the assumption that $f$ is a continuous function on $[0,+\infty)$. In [3], Stuart studies it with the same assumptions as in the present paper, but supposes that $h(0)>0$. In [5], Nistri treats the case $h(p)=0$ for all $p \in[0,1]$ and $k(p)>0$ on $[1,+\infty)$.

\section{General properties of $\boldsymbol{S}^{+}$}

We start this section by giving a theorem concerning the structure of any solution $(u, \lambda)$ in $S^{+}$.

THEOREM 2.1. Let $(u, \lambda) \in S^{+}$, then:

(1) $\lambda>0$ and $u(x)>0$ for all $x \in(0,1)$;

(2) $u^{\prime}(x)>0$ for all $x \in\left[0, \frac{1}{2}\right)$ and $u^{\prime}(x)<0$ for all $x \in\left(\frac{1}{2}, 1\right]$;

(3) $u\left(\frac{1}{2}\right)=\|u\|$ and $u^{\prime}\left(\frac{1}{2}\right)=0$;

(4) if $\|u\|=1$, then $\{x \in[0,1] \mid u(x)=1\}=\left\{\frac{1}{2}\right\}$;

if $\|u\|>1$, there exists $x_{0}$ in $\left(0, \frac{1}{2}\right)$ such that $\{x \in[0,1] \mid u(x)=1\}=\left\{x_{0}, 1-x_{0}\right\}$;

(5) $u(x)=u(1-x)$ for all $x \in[0,1]$.

The proof of this theorem is given in [1].

Let $F(\omega)=\int_{0}^{\omega} f(s) d s$ for $\omega>0$. Since $f(s)>0$ for all $s>0, F$ is a strictly increasing function on $(0,+\infty)$.

Let $g(p)=\sqrt{2} \int_{0}^{p}\{F(p)-F(\omega)\}^{-\frac{1}{2}} d \omega$ for $p>0$. We note that $g(p)<+\infty$ for $p>0$ and $\mathrm{g}$ is a continuous function on $(0,+\infty)$. Moreover $\lim _{n \rightarrow+\infty} g_{n}(p)=g(p)$ for all $p>0$. 
LEMma 2.2 Suppose that $(u, \hat{\lambda}) \in S^{+}$. Then $\lambda^{\frac{1}{2}}=g(\|u\|)$.

Proof. Let us assume that $\|u\|>1$. By Theorem 2.1, there exists a number $x_{0} \in\left(0, \frac{1}{2}\right)$ such that $u\left(x_{0}\right)=1$ and $-u^{\prime \prime}(x)=\lambda f(u(x))$ for all $x \in\left[0, x_{0}\right) \cup\left(x_{0}, \frac{1}{2}\right]$. We obtain

$$
\begin{aligned}
& -\frac{1}{2}\left(u^{\prime}(x)\right)^{2}=\lambda F(u(x))+c_{1} \text { for all } \quad x \in\left[0, x_{0}\right) \\
& -\frac{1}{2}\left(u^{\prime}(x)\right)^{2}=\lambda F(u(x))+c_{2} \text { for all } x \in\left(x_{0}, \frac{1}{2}\right] .
\end{aligned}
$$

Since $u \in C^{1}([0,1])$ and $F$ is continuous on $(0,+\infty)$, it follows that $c_{1}=c_{2}=$ $-\lambda F(\|u\|)$.

Thus $u^{\prime}(x)=\sqrt{2 \lambda}\{F(\|u\|)-F(u(x))\}^{\frac{1}{2}}$ for all $x \in\left[0, \frac{1}{2}\right]$, and consequently $\lambda^{\frac{1}{2}}=g(\|u\|)$.

If $\|u\| \leqq 1$, a similar argument shows that $\lambda^{\frac{1}{2}}=g(\|u\|)$. This completes the proof.

This relationship was introduced in this connection by Laetsch [4].

COROLlaRY 2.3. (1) Suppose that $\left(u_{1}, \lambda_{1}\right)$ and $\left(u_{2}, \lambda_{2}\right)$ are two solutions of problem (1.1) in $S^{+}$such that $\left\|u_{1}\right\|=\left\|u_{2}\right\|$. Then $\lambda_{1}=\lambda_{2}$ and $u_{1}(x)=u_{2}(x)$ on $[0,1]$.

(2) Let $\lambda>0$. Suppose that $\left(u_{1}, \lambda\right)$ and $\left(u_{2}, \lambda\right)$ are two distinct solutions of problem $(1.1)$ in $S^{+}$. Then either $u_{1}(x)<u_{2}(x)$ on $(0,1)$ or $u_{1}(x)>u_{2}(x)$ on $(0,1)$.

Now that all these preliminary results have been obtained, we can state out main existence theorem.

THEOREM 2.4. For any $\rho>0$, there exists exactly one solution of problem (1.1) such that $\|u\|=\rho$.

Proof. Since the unicity is given by Corollary 2.3 , we only need to prove the existence of a solution. Let $\rho>0$, then for any $n \in \mathbb{N}$, there exists $\left(u_{n}, \lambda_{n}\right)$ in $S_{n}$ such that $\left\|u_{n}\right\|=\rho$ and $\lambda_{n}=g_{n}^{2}(\rho)$. Since $\lim _{n \rightarrow+\infty} g_{n}^{2}(\rho)=g^{2}(\rho)$ we have that $\left\{\lambda_{n}\right\}_{n \geqq 1}$ converges and that $\lambda \equiv \lim _{n \rightarrow+\infty} \lambda_{n}=g^{2}(\rho)$. Let $\quad \lambda=\sup \left\{\lambda_{n} \mid n \in \mathbb{N}\right\} \quad$ and $l(\rho)$ $=\sup \{f(t) \mid t \in[0, \rho]\}$, then $\bar{\lambda}<+\infty$ and $l(\rho)>0$.

We thus obtain $\left|u_{n}^{\prime}(x)-u_{n}^{\prime}(y)\right|=\lambda_{n}\left|\int_{x}^{y} f_{n}\left(u_{n}(s)\right) d s\right| \leqq \bar{\lambda}(l(\rho)+1)|x-y|$ for all $x, y$ in $[0,1]$ and all $n \in \mathbb{N}$. If we put $y=\frac{1}{2}$, we have $\left\|u_{n}^{\prime}\right\| \leqq \bar{\lambda}(l(\rho)+1)$ for all $n \in \mathbb{N}$. Therefore, by the Ascoli-Arzelà theorem, there exists a subsequence $\left\{u_{n_{j}}\right\}_{j \geqq 1}$ of $\left\{u_{n}\right\}_{n \geq 1}$ which converges to $u$ in $C^{1}([0,1])$. It follows that $u\left(\frac{1}{2}\right)=\|u\|=\rho, u(0)$ $=u(1)=0$ and $u^{\prime}(x) \geqq 0$ for all $x \in\left[0, \frac{1}{2}\right]$. Since $u(x)=u(1-x)$ for all $x \in[0,1]$, it remains to show that $u^{\prime}(x)=-\lambda \int_{\frac{1}{2}}^{x} f(u(s)) d s$ for all $x \in\left[0, \frac{1}{2}\right]$.

(a) Suppose that $\rho \leqq 1$, then

$$
\begin{aligned}
u^{\prime}(x)=\lim _{j \rightarrow+\infty} u_{n_{j}}^{\prime}(x) & =\lim _{j \rightarrow+\infty}-\lambda_{n_{j}} \int_{\frac{1}{2}}^{x}\left\{h\left(u_{n_{j}}(s)\right)+\left(1 / n_{j}\right)\right\} d s \\
& =-\lambda \int_{\frac{1}{2}}^{x} h(u(s)) d s
\end{aligned}
$$


for all $x \in\left[0, \frac{1}{2}\right]$. Therefore

and we have

$$
\{x \in[0,1] \mid u(x)=1\} \subset\left\{\frac{1}{2}\right\},
$$

$$
\int_{\frac{1}{2}}^{x} h(u(s)) d s=\int_{\frac{1}{2}}^{x} f(u(s)) d s \quad \text { on }\left[0, \frac{1}{2}\right] .
$$

(b) Suppose that $\rho>1$. Then, there exists a number $x_{0}$ in $\left(0, \frac{1}{2}\right)$ such that

$$
\{x \in[0,1] \mid u(x)=1\}=\left\{x_{0}, 1-x_{0}\right\} .
$$

(1) For any $x \in\left(0, x_{0}\right)$, there exists $j_{x} \in \mathbb{N}$ such that $u_{n_{j}}(s)<1$ for all $j \geqq j_{x}$ and all $s \in[0, x]$. Thus,

$$
\begin{aligned}
u^{\prime}(x) & =\lim _{j \rightarrow+\infty}\left[-\lambda_{n_{j}} \int_{0}^{x}\left\{h\left(u_{n_{j}}(s)\right)+\frac{1}{n_{j}}\right\} d s+u_{n_{j}}^{\prime}(0)\right] \\
& =-\lambda \int_{0}^{x} f(u(s)) d s+u^{\prime}(0) \text { for all } x \in\left[0, x_{0}\right) .
\end{aligned}
$$

Since $u^{\prime}$ is a continuous function on $[0,1]$, we have $u^{\prime}(x)=-\lambda \int_{0}^{x} f(u(s)) d s$ $+u^{\prime}(0)$ for all $x \in\left[0, x_{0}\right]$.

(2) A similar argument shows that $u^{\prime}(x)=-\lambda \int_{\frac{1}{2}}^{x} f(u(s)) d s$ on $\left[x_{0}, \frac{1}{2}\right]$.

And it follows from (1) and (2), that $u^{\prime}(x)=-\lambda \int_{\frac{1}{2}}^{x} f(u(s)) d s$ for all $x \in\left[0, \frac{1}{2}\right]$. This completes the proof of this theorem.

Theorem 2.4 allows us to consider the function $\sigma:(0,+\infty) \rightarrow C^{1}([0,1])$ which is defined by: $\left(\sigma(\rho), g^{2}(\rho)\right) \in S^{+}$and $\|\sigma(\rho)\|=\rho$. On $S^{+}$we consider the topology induced from $C^{1}([0,1]) \times[0,+\infty)$.

THEOREM 2.5. The one-to-one map $\psi:(0,+\infty) \rightarrow S^{+}$defined by $\psi(\rho)$ $=\left(\sigma(\rho), g^{2}(\rho)\right)$ is continuous.

An immediate consequence of this last theorem is that $S^{+}$is a continuum in $C^{1}([0,1]) \times[0,+\infty)$ and furthermore $S^{+}$is a continuous curve in $C^{1}([0,1]) \times$ $[0,+\infty)$ which can be parameterized by $\|u\|$.

It follows, from Lemma 2.2 and Theorem 2.4, that the number of solutions of problem (1.1) in $S^{+}$is given, for any $\lambda>0$, by the graph of $g^{2}$. With the purpose of obtaining better information about this graph, we give two different representations of $g^{\prime}$.

\section{First representation of $g^{\prime}$}

We note that, for $p>0$,

where

$$
g(p)=\sqrt{2} p^{\frac{1}{2}} \int_{0}^{1} R(p, t)^{-\frac{1}{2}} d t
$$

$$
R(p, t)=\int_{t}^{1} f(p z) d z \text { for all }(p, t) \in(0,+\infty) \times[0,1] .
$$


Let

$$
\Delta_{p_{0}}= \begin{cases}{\left[\frac{p_{0}}{2}, \bar{p}_{0}\right]} & \text { if } \quad p_{0} \in(0,1) \\ {\left[\bar{p}_{0}, 2 p_{0}\right]} & \text { if } \quad p_{0} \in(1,+\infty) .\end{cases}
$$

Where $\bar{p}=\left(1+p_{0}\right) / 2$ for all $p_{0} \in(0,+\infty)$.

LEMMA 2.6. For all $p_{0} \in(0,1) \cup(1,+\infty)$, there exists a positive constant $d\left(p_{0}\right)$ such that

$$
R(p, t)^{-\frac{3}{2}}\left|\int_{0}^{1} f^{\prime}(p z) z d z\right| \leqq d\left(p_{0}\right)(1-t)^{-\frac{1}{2}} \text { for all }(p, t) \in \Delta_{p_{0}} \times[0,1)
$$

LEMMA 2.7. Let $T(p)=\int_{0}^{1} R(p, t)^{-\frac{1}{2}} d t$. Then $T$ is continuously differentiable on $(0,1) \cup(1,+\infty)$. Furthermore

$$
T^{\prime}(p)=-\frac{1}{2} \int_{0}^{1} R(p, t)^{-\frac{3}{2}} \int_{t}^{1} f^{\prime}(p z) z d z d t \quad \text { if } \quad p \in(0,1)
$$

and

$$
T^{\prime}(p)=-\frac{1}{2} \int_{0}^{1} R(p, t)^{-\frac{3}{2}} \int_{t}^{1} f^{\prime}(p z) z d z d t-\frac{1}{2} p^{-2}\{k(1)-h(1)\} \int_{0}^{1 / p} R(p, t)^{-\frac{3}{2}} d t
$$

if $p \in(1,+\infty)$.

Proof. (1) If $p \in(0,1)$, it follows by using Lemma 2.6, that

$$
T^{\prime}(p)=\frac{d}{d p} \int_{0}^{1} R(p, t)^{-\frac{1}{2}} d t=\int_{0}^{1} \frac{\partial R(p, t)^{-\frac{1}{2}}}{\partial p} d t=-\frac{1}{2} \int_{0}^{1} R(p, t)^{-\frac{3}{2}} \int_{t}^{1} f^{\prime}(p z) z d z d t
$$

$T^{\prime}$ is continuous on $(0,1)$.

(2) If $p>1$, then

$$
T(p)=\int_{0}^{1} R(p, t)^{-\frac{1}{2}} d t=\int_{0}^{1 / p} R(p, t)^{-\frac{1}{2}} d t+\int_{1 / p}^{1} R(p, t)^{-\frac{1}{2}} d t .
$$

And it follows, by using Lemma 2.6, that

$$
T^{\prime}(p)=-\frac{1}{2} \int_{0}^{1} R(p, t)^{-\frac{3}{2}} \int_{t}^{1} f^{\prime}(p z) z d z d t-\frac{1}{2} p^{-2}\{k(1)-h(1)\} \int_{0}^{1 / p} R(p, t)^{-\frac{3}{2}} d t .
$$

$T^{\prime}$ is continuous on $(1,+\infty)$.

Now, we are able to give the first representation of $g^{\prime}$. 
COROLlaRY 2.8. $g$ is continuously differentiable on $(0,1) \cup(1,+\infty)$. Furthermore

$$
g^{\prime}(p)=\sqrt{2}\left\{\frac{1}{2} p^{-\frac{1}{2}} \int_{0}^{1} R(p, t)^{-\frac{1}{2}} d t-\frac{1}{2} p^{\frac{1}{2}} \int_{0}^{1} R(p, t)^{-\frac{3}{2}} \int_{t}^{1} f^{\prime}(p z) z d z d t\right\} \quad \text { if } \quad p \in(0,1)
$$

and

$$
\begin{aligned}
g^{\prime}(p)= & \sqrt{2}\left\{\frac{1}{2} p^{-\frac{1}{2}} \int_{0}^{1} R(p, t)^{-\frac{1}{2}} d t-\frac{1}{2} p^{\frac{1}{2}} \int_{0}^{1} R(p, t)^{-\frac{3}{2}} \int_{t}^{1} f^{\prime}(p z) z d z d t\right. \\
& \left.-\frac{1}{2} p^{-\frac{3}{2}}\{k(1)-h(1)\} \int_{0}^{1 / p} R(p, t)^{-\frac{3}{2}} d t\right\} \text { if } p \in(1,+\infty) .
\end{aligned}
$$

\section{Second representation of $g^{\prime}$}

LEMMA 2.9.

$$
\begin{aligned}
g^{\prime}(p)= & \sqrt{2} f(p)\left\{-\frac{1}{2} \int_{0}^{\frac{1}{2}}\{F(p)-F(\omega)\}^{-\frac{3}{2}} d \omega+\left\{\frac{1}{k(1)}-\frac{1}{h(1)}\right\}\{F(p)-F(1)\}^{-\frac{1}{2}}\right. \\
& \left.+\frac{1}{h\left(\frac{1}{2}\right)}\left\{F(p)-F\left(\frac{1}{2}\right)\right\}^{-\frac{1}{2}}-\int_{\frac{1}{2}}^{p}\{F(p)-F(\omega)\}^{-\frac{1}{2}} \frac{f^{\prime}(\omega)}{f^{2}(\omega)} d \omega\right\} \text { for all } p>1 .
\end{aligned}
$$

Proot. Since

$$
\begin{aligned}
g(p)= & \sqrt{2}\left\{\int_{0}^{1}\{F(p)-F(\omega)\}^{-\frac{1}{2}} d \omega+\frac{2}{k(1)}\{F(p)-F(1)\}^{\frac{1}{2}}\right. \\
& \left.-2 \int_{1}^{p}\{F(p)-F(\omega)\}^{\frac{1}{2}} \frac{f^{\prime}(\omega)}{f^{2}(\omega)} d \omega\right\} \text { for all } p>1
\end{aligned}
$$

by differentiation we obtain the assertion.

COROllary 2.10 .

$$
\lim _{p \rightarrow 1^{+}} g^{\prime}(p)=\left\{\begin{array}{lll}
+\infty & \text { if } & h(1)>k(1) \\
-\infty & \text { if } & h(1)<k(1)
\end{array}\right.
$$

Having shown, in Theorem 2.4, that there is exactly one solution of problem (1.1) in $S^{+}$for each value of $\|u\|$, let us now ask for which values of $\lambda>0$ there is a solution. The next lemma helps us to answer this question.

LEMMA 2.11. Suppose that $\lim _{p \rightarrow+\infty} p^{-1} f(p)=0$. Then $\lim _{p \rightarrow+\infty} g(p)=+\infty$.

Proof. Let $n \in \mathbb{N}-\{1\}$. Then there exists a positive number $p_{n}$ such that

$$
\{F(p)-F(\omega)\} \leqq \frac{1}{2 n}\left(p^{2}-\omega^{2}\right) \text { for all } p \geqq \omega \geqq p_{n} \text {. }
$$


Let $\bar{p}_{n}=n p_{n}$, then if $p \geqq \bar{p}_{n}$ we obtain

$$
g(p) \geqq 2 \sqrt{n} \int_{n / n}^{p} \frac{d \omega}{\sqrt{\left(p^{2}-\omega^{2}\right)}}=2 \sqrt{n}\left\{\frac{\pi}{2}-\operatorname{Arcsin} \frac{1}{n}\right\} \geqq 2 \sqrt{n} .
$$

Therefore $\lim _{p \rightarrow+\infty} g(p)=+\infty$.

THEOREM 2.12. Suppose that $\lim _{p \rightarrow+\infty} p^{-1} f(p)=0$ and that $h(1)<k(1)$. Then there exist numbers $\lambda_{1}$ and $\lambda_{2}$ with $0<\lambda_{1}<\lambda_{2}$ such that, for each $\lambda \in\left(\lambda_{1}, \lambda_{2}\right)$, there are at least two distinct solutions of problem (1.1) in $S^{+}$.

Proof. Since $g^{2}$ is continuous on $[1,+\infty), \lim _{p \rightarrow+\infty} g(p)=+\infty$ (Lemma 2.11) and $\lim _{p \rightarrow 1}+g^{\prime}(p)=-\infty$ (Corollary 2.10), there exists a number $\rho_{1}>1$ such that $0<\lambda_{1}=\min \left\{g^{2}(p) \mid p \geqq 1\right\}=g^{2}\left(\rho_{1}\right)<g^{2}(1)=\lambda_{2}$. Therefore, for any $\lambda \in\left(\lambda_{1}, \lambda_{2}\right)$, there exist two numbers $\rho$ and $\bar{\rho}$ with $1<\rho<\rho_{1}<\bar{\rho}$ such that $g^{2}(\rho)=g^{2}(\bar{\rho})=\hat{\lambda}$. It follows that $(\sigma(\rho), \lambda)$ and $(\sigma(\bar{\rho}), \lambda)$ are two distinct solutions of problem (1.1) in $S^{+}$. This completes the proof.

\section{Study of $S^{+}$when $h^{\prime}(0)>0$}

Let $\mathscr{C}$ denote the subset of $C^{1}([0,1]) \times[0,+\infty)$ defined by $\mathscr{C}=S^{+} \cup\left\{\left(0, \pi^{2} / \alpha\right)\right\}$ where $\alpha=\lim _{p \rightarrow 0^{+}} p^{-1} f(p)=h^{\prime}(0)$. On $\mathscr{C}$ we consider the topology induced from $C^{1}([0,1]) \times[0,+\infty)$.

Let

$$
\tilde{g}(p)=\left\{\begin{array}{lll}
g(p) & \text { if } \quad p>0 \\
\frac{\pi}{\sqrt{\alpha}} & \text { if } \quad p=0
\end{array} \quad \text { and } \quad \tilde{\psi}(p)= \begin{cases}\psi(p) & \text { if } p>0 \\
\left(0, \frac{\pi^{2}}{\alpha}\right) & \text { if } p=0 .\end{cases}\right.
$$

Then it is easy to see that $\tilde{g}:[0,+\infty) \rightarrow(0,+\infty)$ is a continuous function and hence $\tilde{\psi}:[0,+\infty) \rightarrow \mathscr{C}$ is a continuous function. It immediately follows that $\mathscr{C}$ is a continuum in $C^{1}([0,1]) \times[0,+\infty)$, and furthermore $\mathscr{C}$ is a continuous curve in $C^{1}([0,1] \times[0,+\infty)$ which can be parametrized by $\|u\|$.

PROPOSITION 3.1. Suppose that $\lim _{p \rightarrow+\infty} p^{-1} f(p)=0$. Then, for any $i \geqq \pi^{2} / \alpha$, there is at least one solution of problem (1.1) in $\mathscr{C}$.

Proof. We see from Lemma 2.11 that $\lim _{p \rightarrow+\infty} \tilde{g}(p)=+\infty$. The result follows immediately from this and the continuity of $\tilde{g}$ on $[0,+\infty)$.

PROPOSITION 3.2. Suppose that $\liminf _{p \rightarrow+\infty} p^{-1} f(p)=\beta>0$. Then, there exists a positive constant $\gamma$ such that $f(p) \geqq \gamma p$ for all $p \geqq 0$ and $\{i \geqq 0 \mid$ there exists $(u, \lambda) \in \mathscr{C}\} \subset\left(0, \pi^{2} / \gamma\right]$.

Proof. Since $\liminf _{p \rightarrow+\infty} p^{-1} f(p)=\beta>0$, there exist two positive numbers $\bar{\beta}$ and $p_{0}$ such that $f(p) \geqq \beta p$ for all $p \geqq p_{0}$. Since $\lim _{p \rightarrow 0^{+}} p^{-1} f(p)=\alpha>0$, the number 
$m=\inf \left\{p^{-1} f(p) \mid p \in\left(0, p_{0}\right]\right\}$ is positive. Let $\gamma=\min \{\bar{\beta}, m\}$, then $f(p) \geqq \gamma p$ for all $p$ $\geqq 0$.

If $(u, \lambda) \in \mathscr{C}$ with $\|u\| \neq 0$, then $\{F(\|u\|)-F(\omega)\} \geqq \gamma / 2\left(\|u\|^{2}-\omega^{2}\right)$ for all $\omega \in[0,\|u\|]$, and we obtain

$$
\sqrt{\lambda}=\sqrt{2} \int_{0}^{\|u\|}\{F(\|u\|)-F(\omega)\}^{-\frac{1}{2}} d \omega \leqq \frac{\pi}{\sqrt{\gamma}}
$$

If $(u, \lambda) \in \mathscr{C}$ with $\|u\|=0$, then $\lambda=\left(\pi^{2} / \alpha\right) \leqq\left(\pi^{2} / \gamma\right)$.

This completes the proof of this proposition.

Now, we study the structure of $\mathscr{C}$ under the assumption (A): $f(p) \geqq p f^{\prime}(p)$ for all $p \in[0,1) \cup(1,+\infty)$ and $h(1)>k(1)$. Under this assumption, $p^{-1} f(p)$ is a nonincreasing function on $[0,1) \cup(1,+\infty)$. Let $m=\lim _{p \rightarrow+\infty} p^{-1} f(p)$, then it is easy to show that

$$
\xi=\lim _{p \rightarrow+\infty} \tilde{g}(p)=\left\{\begin{array}{lll}
\frac{\pi}{\sqrt{m}} & \text { if } & m \neq 0 \\
+x & \text { if } & m=0 .
\end{array}\right.
$$

THEOREM 3.3. Let $a \in[0,1]$. Suppose that:

(i) fsatisfies assumption (A),

(ii) $f(p)=\alpha p$ for all $p \in(0, a)$,

(iii) $f(p) \neq \alpha p$ for all $p \in(a, 1)$,

then

(1) $\left\{(u, \lambda) \in \mathscr{C} \mid \lambda \in\left[0, \pi^{2} / \alpha\right) \cup\left[\xi^{2},+\infty\right)\right\}=\varnothing$,

(2) $\left\{(u, \lambda) \in \mathscr{C} \mid \lambda=\pi^{2} / \alpha\right\}=\left\{\left(\gamma \sin \pi, \pi^{2} / \alpha\right) \mid \gamma \in[0, a]\right\}$,

(3) for any $\lambda \in\left(\pi^{2} / \alpha, \xi^{2}\right)$, there exists exactly one solution of problem (1.1) in $\mathscr{C}$.

Proof. By assumption (i), we have that $\int_{t}^{1} f^{\prime}(p z) z d z \leqq p^{-1} R(p, t)$ for all $(p, t) \in(0,+\infty) \times[0,1]$. Suppose that $a \neq 1$, then for all $p \in(a, 1)$ there exist two numbers $t_{p}$ and $\bar{t}_{p}$ in $(0,1)$ with $t_{p}<\bar{t}_{p}$ such that $\int_{t}^{1} f^{\prime}(p z) z d z<p^{-1} R(p, t)$ for all $t \in\left(t_{p}, \bar{t}_{p}\right)$. And we obtain, by the first representation of $g^{\prime}$, that $\tilde{g}^{\prime}(p)>0$ for all $p \in(a, 1) \cup(1,+\infty)$. Thus, this theorem becomes an immediate consequence of Lemma 2.2 and Theorem 2.4 .

In the same way, we can study the structure of $\mathscr{C}$ under the following assumption (B): $f(p) \leqq p f^{\prime}(p)$ for all $p \in[0,1) \cup(1,+\infty)$ and $h(1)<k(1)$. In this case. $p^{-1} f(p)$ is a non-decreasing function on $[0,1) \cup(1,+\infty)$. Let $r=\lim _{p \rightarrow+\infty} p^{-1} f(p)$, then it is easy to show that

$$
\xi=\lim _{p \rightarrow+\infty} \tilde{g}(p)=\left\{\begin{array}{ccc}
\frac{\pi}{\sqrt{r}} & \text { if } & r \in[0,+\infty) \\
0 & \text { if } & r=+\infty .
\end{array}\right.
$$


TheOREM 3.4. Let $a \in[0,1]$. Suppose that:

(i) $f$ satisfies assumption (B),

(ii) $f(p)=\alpha p$ for all $p \in(0, a)$,

(iii) $f(p) \neq \alpha p$ for all $p \in(a, 1)$,

then

(1) $\left\{(u, \lambda) \in \mathscr{C} \mid \lambda \in\left[0, \xi^{2}\right] \cup\left(\pi^{2} / \alpha,+\infty\right)\right\}=\varnothing$,

(2) $\left\{(u, \lambda) \in \mathscr{C} \mid \lambda=\pi^{2} / \alpha\right\}=\left\{\left(\gamma \sin \pi, \pi^{2} / \alpha\right) \mid \gamma \in[0, a]\right\}$,

(3) for any $\lambda \in\left(\xi^{2}, \pi^{2} / \alpha\right)$, there exists exactly one solution of problem (1.1) in $\mathscr{C}$.

In our next theorem, we give an example of a function $f$ for which there are, for some $\lambda>0$, at least three distinct solutions of problem (1.1) in $\mathscr{C}$.

THEOREM 3.5. Let $a \in[0,1)$. Suppose that

(i) $f(p) \geqq p f^{\prime}(p)$ for all $p \in[0,1)$,

(ii) $f(p)=\alpha p \quad$ for all $p \in[0, a]$,

(iii) $f(p) \neq \alpha p \quad$ for all $p \in(a, 1)$,

(iv) $h(1)<k(1)$,

(v) $\lim _{p \rightarrow+\infty} p^{-1} f(p)=0$.

Then, there exist numbers $\lambda_{1}$ and $\lambda_{2}$ with $0<\lambda_{1}<\lambda_{2}$ such that for any $\lambda \in\left(\lambda_{1}, \lambda_{2}\right)$ there exist at least three distinct solutions of problem (1.1) in $\mathscr{C}$.

Proof. Since $\tilde{g}^{2}$ is continuous on $[1,+\infty), \lim _{p \rightarrow+\infty} \tilde{g}(p)=+\infty$ (Lemma 2.11) and $\lim _{p \rightarrow 1}+\tilde{g}^{\prime}(p)=-\infty$ (Corollary 2.10 ), we have that $\bar{\lambda}=\min \left\{\tilde{g}^{2}(p) \mid p \geqq 1\right\}$ exists and that $0<\bar{\lambda}<\tilde{g}^{2}(1)=\lambda_{2}$. We obtain from assumptions (i) and (iii) that $\lambda_{2}>\pi^{2} / x$. Let $\lambda_{1}=\max \left\{\bar{\lambda}_{,}\left(\pi^{2} / \alpha\right)\right\}$, then $0<\lambda_{1}<\lambda_{2}$ and there exists a number $\bar{\rho}_{1}>1$ such that $\tilde{g}^{2}\left(\bar{\rho}_{1}\right)=\lambda_{1}$. Let $i \in\left(\lambda_{1}, \lambda_{2}\right)$. Since $\tilde{g}^{2}$ is a continuous function on $[0,+\infty)$ and $\lim _{p \rightarrow+\infty} \tilde{g}^{2}(p)=+\infty$ (Lemma 2.11), there exist numbers $\rho_{1}, \rho_{2}$ and $\rho_{3}$ with $0<\rho_{1}$ $<1<\rho_{2}<\bar{\rho}_{1}<\rho_{3}$ such that $\lambda=\tilde{g}^{2}\left(\rho_{1}\right)=\tilde{g}^{2}\left(\rho_{2}\right)=\tilde{g}^{2}\left(\rho_{3}\right)$. Therefore $\left(\sigma\left(\rho_{1}\right), \lambda\right)$, $\left(\sigma\left(\rho_{2}\right), \lambda\right)$ and $\left(\sigma\left(\rho_{3}\right), \lambda\right)$ are three distinct solutions of problem (1.1) in $\mathscr{C}$.

\section{Study of $S^{+}$when $h^{\prime}(0)=0$}

Since $h^{\prime}(0)=0$, it follows immediately that $\lim _{p \rightarrow 0^{+}} g(p)=+\infty$.

PROPOSITION 4.1. There exists a number $\hat{\lambda} \geqq 0$ such that for any $\lambda>\hat{\lambda}$ there is at least one solution of problem (1.1) in $S^{+}$.

PROPOSITION 4.2. Suppose that $\lim _{p \rightarrow+\infty} p^{-1} f(p)=0$. Then, there exists a positive number $\lambda_{1}$ such that for any $\lambda>\lambda_{1}$ the problem (1.1) has at least two distinct solutions in $S^{+}$, for $\lambda=\lambda_{1}$ at least one and for $\lambda \in\left[0, \lambda_{1}\right)$ none.

Proof. Since $\lim _{p \rightarrow 0^{+}} g^{2}(p)=+\infty, \lim _{p \rightarrow+\infty} g^{2}(p)=+\infty$ (Lemma 2.11) and $g^{2}$ is a continuous function on $(0,+\infty), \lambda_{1}=\min \left\{g^{2}(p) \mid p>0\right\}$ exists and for any 
$\lambda>\lambda_{1}>0$ there are numbers $\rho, \rho_{1}$ and $\bar{\rho}$ with $0<\rho<\rho_{1}<\bar{\rho}$ such that $g^{2}\left(\rho_{1}\right)=\lambda_{1}$ and $g^{2}(\rho)=g^{2}(\bar{\rho})=\lambda$. Thus, $(\sigma(\rho), \lambda)$ and $(\sigma(\bar{\rho}), \lambda)$ are two distinct solutions of problem (1.1) in $S^{+}$. Furthermore, $\left(\sigma\left(\rho_{1}\right), \hat{\lambda}_{1}\right)$ is also a solution of problem (1.1) in $S^{+}$. This completes the proof of this proposition.

Now, we study the structure of $S^{+}$under assumption (B): $f(p) \leqq p f^{\prime}(p)$ for all $p \in[0,1) \cup(1,+\infty)$ and $h(1)<k(1)$. In this case, $p^{-1} f(p)$ is a non-decreasing function on $(0,1) \cup(1,+\infty)$. Let $m=\lim _{p \rightarrow+\infty} p^{-1} f(p)$, then

$$
\xi=\lim _{p \rightarrow+\infty} \tilde{g}(p)=\left\{\begin{array}{lll}
\frac{\pi}{\sqrt{m}} & \text { if } & m \in(0,+\infty) \\
0 & \text { if } & m=+\infty
\end{array} .\right.
$$

THEOREM 4.3. Suppose that f satisfies assumption (B). Then

(1) $\left\{(u, \lambda) \in S^{+} \mid \lambda \in\left[0, \xi^{2}\right]\right\}=\varnothing$,

(2) for any $\lambda>\xi^{2}$, there exists exactly one solution of problem (1.1) in $S^{+}$.

\section{References}

1 C. A. Stuart. Differential equations with discontinuous nonikinearities. Arch. Rational Mech. Anal. $63(1976), 59-75$.

2 C. A. Stuart. Boundary-value problems with discontinuous non-linearities. Proc. Conf. Diff. Equations, Dundee 1976. Lecture Notes in Mathematics 564, 472-484 (Berlin: Springer, 1977).

3 C. A. Stuart. The number of solutions of boundary-value problems with discontinuous nonlinearities. Arch. Rational Mech. Anal. 66 (1977), 225-235.

4 T. Laetsch. The number of solutions of a non-linear two point boundary-value problem. Indiana Univ. Math. J. 20 (1970), 1-13.

5 P. Nistri. Positive solutions of a non-linear eigenvalue problem with discontinuous non-linearity. Proc. Roy. Soc. Edinburgh Sect. A 83 (1979), 133-145.

(Issued 25 January 1982) 